\title{
Force and Moment Measurements on a Free-Flying Capsule Model in a High-Enthalpy Shock Tunnel
}

\author{
Stuart J. Laurence ${ }^{1}$, Jan Martinez Schramm ${ }^{2}$ and Klaus Hannemann ${ }^{3}$ \\ German Aerospace Center (DLR), Göttingen 37073, Germany
}

\begin{abstract}
Experiments to measure the forces and moments acting on a blunted-cone capsule model are carried out in the High-Enthalpy Shock Tunnel, Göttingen (HEG). A free-flying arrangement is employed, whereby the model is initially suspended by weak threads that are detached by the arrival of the flow, allowing unrestrained flight during the steady test time. High-speed shadowgraphy is used to capture the model motion. Induced forces and moments are measured using both internally mounted accelerometers and visualizationbased tracking techniques. Improvements to the visualization-based techniques allow the elimination of several previously identified sources of error. Measurements are performed over a range of angles of attack, and excellent agreement is obtained between the techniques. The precision of the visualization-based results is such that variation in the free-stream conditions is the dominant source of uncertainty in the drag coefficient, and is comparable to or larger than the standard error in the lift acceleration for angles of attack above approximately $6^{\circ}$. When applied to pitching measurements, all techniques suffer from large standard errors, but the visualization-based techniques show the capability to outperform accelerometer measurements.
\end{abstract}

\section{Introduction}

$\mathrm{T}$ HE accurate measurement of aerodynamic forces and moments experienced by models in high-enthalpy wind tunnels has traditionally been a challenging task ${ }^{1}$. The short measurement times intrinsic to these facilities mean that stress equilibrium typically cannot be established within the model and supporting sting within the test duration, making measurement through conventional force balance techniques impossible. Modifications to force balances to account for shorter test times, either through the interpretation of unsteady stress waves ${ }^{2}$ or by using acceleration compensation $^{3}$, have been proposed and successfully implemented in some cases. An elegantly simple alternative to such methods is to allow the model to fly freely in the flow and to determine the forces from the resulting motion. For such free-flying models, the short measurement time of impulse facilities is in some ways an advantage, since the changes in attitude or position of the model, being proportional to the square of the test time, can typically be neglected despite the large accelerations encountered. Additional advantages of free-flight techniques are the flexibility of model design, and the ability to do away with the sting support and accompanying sting/base-drag interference. The induced accelerations can be either directly measured using internally mounted accelerometers ${ }^{4,5}$ or derived from displacements inferred from a visual record of the model trajectory ${ }^{6,7}$. Recent advances have enabled considerable refinements to both of these measurement methods: for example, the development of a miniature high-speed data-recorder enabled accelerometer measurements to be made on a blunted cone with no interference from supporting structures ${ }^{8}$; and high-speed digital cinematography combined with highly accurate image-processing techniques ${ }^{9,10}$ has allowed the determination of model displacements with an order-of-magnitude improvement in precision over earlier analog-film-based measurements.

For such free-flight techniques, an important consideration is the manner in which the model is initially positioned and/or released into the flow: the ideal method would combine precise placement with rapid release and minimal subsequent aerodynamic interference. Various means devised by earlier researchers to satisfy these requirements are summarized by Bernstein ${ }^{1}$, and this discussion remains substantially relevant today. One notable subsequent development has been the magnetic release system employed by Tanno et al. ${ }^{8}$, which combined a high

\footnotetext{
${ }^{1}$ Research Staff, Institute of Aerodynamics and Flow Technology, Spacecraft Department, Bunsenstraße 10, AIAA Member.

${ }^{2}$ Research Staff, Institute of Aerodynamics and Flow Technology, Spacecraft Department, Bunsenstraße 10.

${ }^{3}$ Head, Spacecraft Department, Institute of Aerodynamics and Flow Technology, Bunsenstraße 10, AIAA Member.
} 
degree of repeatability with entirely negligible flow interference when applied to a large $(\sim 20 \mathrm{~kg})$ blunted-cone model. However, weak suspension techniques that rely on the flow itself to release the model still enjoy advantages in terms of flexibility and simplicity, provided concerns regarding the reliability of release and the accuracy of initial positioning can be addressed.

Here we describe a set of experiments in which force and moment measurements were carried out on a capsule model in the HEG reflected-shock wind tunnel. In HEG, we are currently moving away from stress-wave-based methods (e.g., ref. 11) towards non-intrusive free-flight measurements, and it is desired to obtain reference data to verify the capabilities of recently developed visualization-based measurement techniques ${ }^{10,12}$. A free-flying configuration incorporating internally mounted accelerometers was thus developed and tested. A weak-thread suspension technique was employed, relying on the arrival of the flow itself to detach the supports and eliminating the need for a sophisticated timed release mechanism. High-speed focused shadowgraphy sequences were captured of the model motion, and the accelerations determined using two visualization-based tracking techniques were compared with the accelerometer measurements. For the visualization-based techniques, several improvements were made to eliminate known sources of uncertainty.

\section{Experimental Facility and Configuration}

The facility employed for all experiments described herein was the HEG (High-Enthalpy shock tunnel, Göttingen) of the German Aerospace Center (DLR). The HEG is a reflected-shock tunnel, capable of producing flows over a wide range of stagnation pressures and total enthalpies. A schematic of the facility is shown in Fig. 1; further information on the operating principles and conditions achievable in HEG is provided in Refs. 13 and 14. Briefly, to initiate a test run, compressed air is used to accelerate a free piston down the compression tube, which is filled with a mixture of helium and argon. A primary diaphragm initially separates the compression tube from the shock tube, containing the test gas. When the pressure in front of the piston reaches a sufficiently high level, the primary diaphragm bursts, sending a strong shock wave down the shock tube. This shock reflects from the far end of the shock tube, rupturing the secondary (mylar) diaphragm which separates the tunnel nozzle and test section, initially under vacuum, from the shock tube. The reflected shock also decelerates the test gas in the shock tube to stagnation conditions, forming the reservoir for the subsequent expansion through the hypersonic nozzle and into the test section. Steady test flow conditions typically persist for up to several milliseconds; the test time is terminated either by the arrival of the expansion wave from the primary diaphragm burst, or by contamination of the test flow by the driver (compression tube) gas.

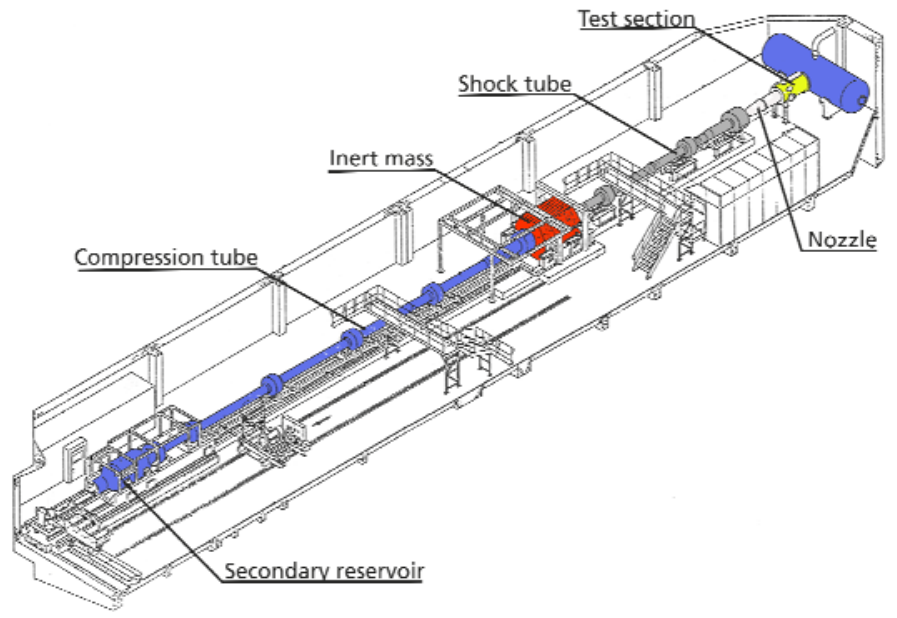

Figure 1: Schematic of the HEG reflected-shock wind tunnel, with the major components labeled.

The test condition employed here was a low-enthalpy air condition (HEG Condition XIII), approximately simulating Mach-8 flight at $28 \mathrm{~km}$ altitude. Typical reservoir and free-stream conditions are provided in table 1 . The stagnation temperature is calculated from the measured reservoir pressure and shock speed using the ESTC code. The free-stream conditions are calculated using the DLR TAU $\operatorname{code}^{15}$ and have been validated by extensive calibration measurements. Figure 2 shows reservoir and Pitot pressures from an experiment in the present investigation. The Pitot pressure, measured using a probe mounted in the test section (see fig. 3), has been scaled so 
that its development can be directly compared to that of the reservoir pressure. The time-axis of the reservoir pressure has been shifted to account for the passage of the flow through the nozzle; $t=0$ corresponds here to the instant of shock reflection from the shock-tube end wall. Following the establishment of the flow, which requires $\sim 3$ $\mathrm{ms}$, the quasi-steady test conditions (the duration of which is indicated by vertical black lines) persist for approximately $2 \mathrm{~ms}$, before the pressures begin to drop gradually due to the arrival of the expansion waves from the primary diaphragm rupture. Following the first $\sim 1 \mathrm{~ms}$ of flow development, the Pitot and reservoir pressures are seen to mirror each other closely.

\begin{tabular}{|l|l|l|l|l|l|l|}
\hline$p_{0}, \mathrm{MPa}$ & $T_{0}, \mathrm{~K}$ & $u_{\infty}, \mathrm{m} / \mathrm{s}$ & $\rho_{\infty}, \mathrm{kg} / \mathrm{m}^{3}$ & $p_{\infty}, \mathrm{Pa}$ & $T_{\infty}, \mathrm{K}$ & $M_{\infty}$ \\
\hline 18.3 & 2720 & 2410 & 0.0275 & 2130 & 268 & 7.34 \\
\hline
\end{tabular}

Table 2: Reservoir (subscript 0 ) and free-stream (subscript $\infty$ ) conditions for the HEG test condition employed in the present experiments.

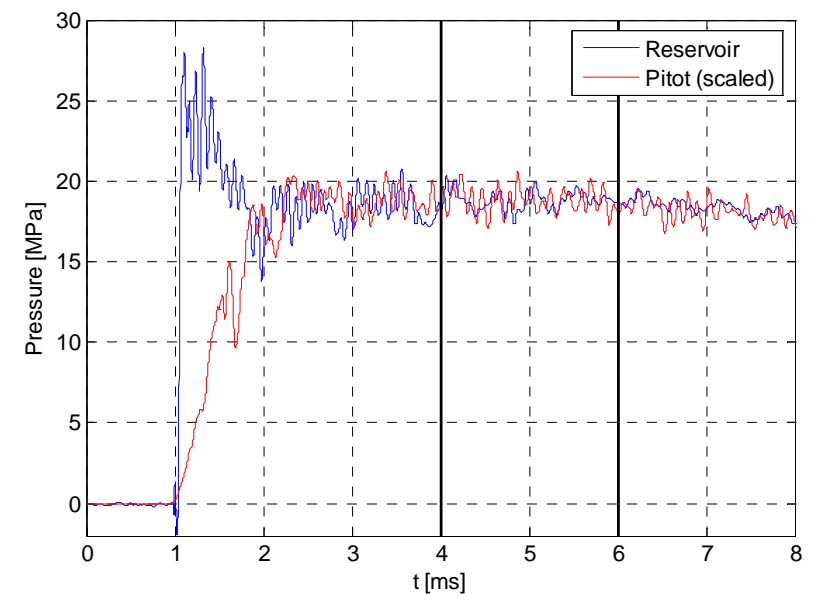

Figure 2. Measured reservoir and Pitot pressures (the latter scaled) during a typical experiment. The reservoir trace has been shifted to account for the time taken for the flow to pass through the nozzle. The quasi-steady test time $(4.0-6.0 \mathrm{~ms})$ is indicated by the solid vertical lines.

The experimental configuration for the present measurements is shown in Fig. 3. The capsule model is $18 \mathrm{~cm}$ in diameter, with a forebody consisting of a blunt spherical nose blended to a $70^{\circ}$ half-angle cone. The model is constructed of stainless steel and has a mass of $3.1 \mathrm{~kg}$. The internal design was such as to allow for the inclusion of embedded accelerometers and associated cabling (detailed in the next section). A short, flared bulb-like protuberance was attached to the rear of the model, forming part of the catcher mechanism to terminate the model motion. The other part of this mechanism consisted of a housing fixed to a conventional sting, inside of which the model protuberance was placed prior to the experiment. The housing allowed for a model motion of approximately $15 \mathrm{~mm}$ in the axial direction and several millimeters in the lateral direction and prevented the model from falling to the test section floor following the conclusion of the test. The model was suspended prior to each experiment by two lengths of thread in V-arrangements. The front thread passed inside the model and was responsible principally for supporting the model weight as well as controlling the model height: nylon thread was employed, specifically weakened at the junctions with the model body so that it would break on flow arrival without leaving significant excrescences. The rear thread was looped under the rear protuberance and was used primarily to control the incidence angle; since little weight was supported by this thread, weaker cotton material could be used.

The two threads passed up to a positioning plate attached to the test-section roof (shown in Fig. 3), and each was looped over a guiding block attached to a travelling screw. The model height and incidence angle could thus be adjusted independently of one another. The threads exhibited the tendency to stretch gradually once the model had been suspended; however, if the model was left hanging overnight prior to the experiment, the change in incidence angle from the time the test section was closed until firing of the facility was typically less than $0.1^{\circ}$. The yaw alignment of the model was checked by ensuring that the suspension threads were coincident both visually and in recorded images. As the threads were $0.5 \mathrm{~mm}$ in diameter and separated by roughly $140 \mathrm{~mm}$, provided they appeared as a single thread, the model was aligned in the yaw axis to within $0.2^{\circ}$. 


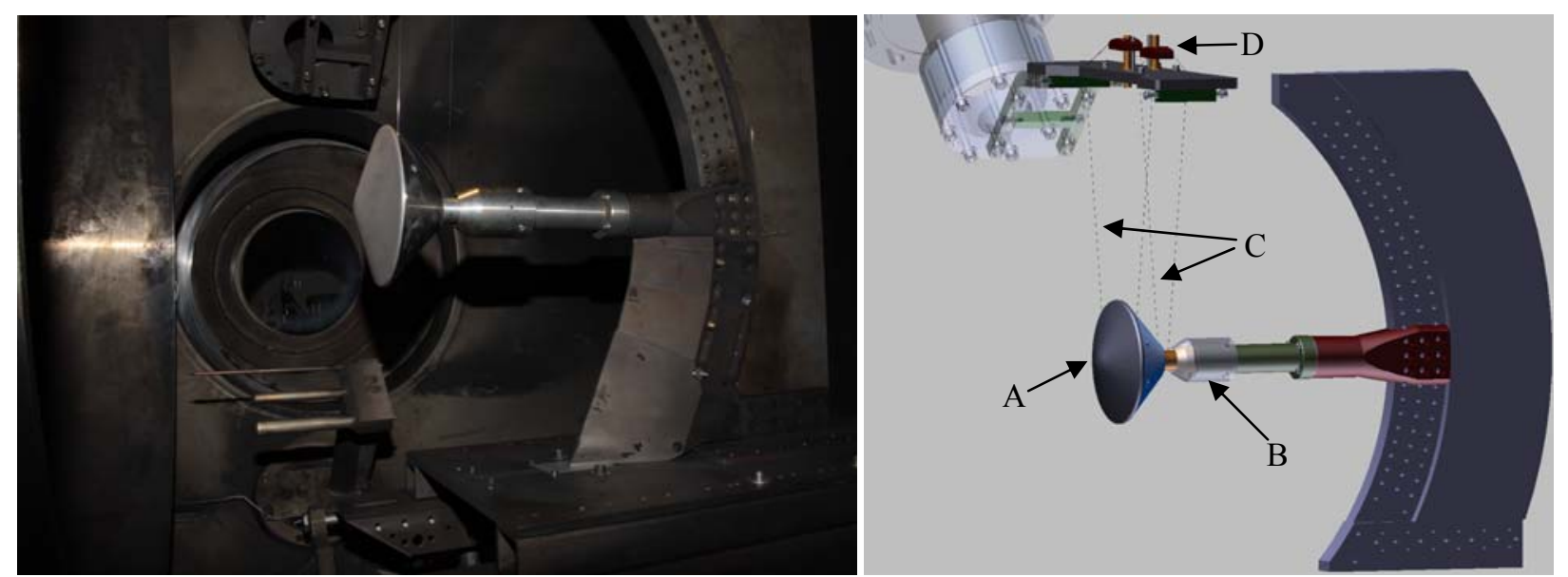

Figure 3. Photograph (left) and schematic (right) of the experimental setup inside the HEG test section: (A) capsule model; (B) housing of catcher mechanism; (C) suspension threads; (D) height/incidence adjustment mechanism on positioning plate. The Pitot/static-pressure probe is visible in the photograph to the lower left of the capsule model.

\section{Measurement Techniques}

Inside the model was fixed a circular instrumentation plate with five attachment points for accelerometers, allowing measurements of up to five components of the motion (three translational and two rotational). Measurements of the yaw and roll components showed both of these to be negligible, as expected. The accelerometers employed were MMF KS95B10 piezoelectric-type transducers; this sensor type has a sensitivity of approximately $10 \mathrm{mV} / \mathrm{g}$ and a resonant frequency above $40 \mathrm{kHz}$. To avoid the excitation of such high frequency components, each accelerometers was mechanically damped by fixing a PVC plate between the sensor and the instrumentation plate. The instrumentation cables passed from the accelerometers through the catcher mechanism and down to the feed-through plate at the test-section floor; from here, the signals were fed into PCB signal conditioners and were recorded at $1 \mathrm{MHz}$ with the HEG data-acquisition system. The influence of the cables on the experienced forces was determined to be negligible.

For visualization we employed a focused shadowgraph setup, consisting of a conventional Z-arm Schlieren arrangement with the knife-edge removed ${ }^{16}$. The use of focused shadowgraphy minimizes the sensitivity to density gradients, reducing the influence of visualized flow features on the accuracy of the tracking technique to be described shortly. The camera used for the bulk of these experiments was a Shimadzu HPV-1 with a fixed resolution of 312x260 pixels; the frame-rate and exposure time were $16 \mathrm{kfps}$ and $8 \mu \mathrm{s}$, respectively. The light source employed was a Cavilux Smart pulsed-diode laser. This device, having been designed specifically for flow visualization purposes, has several advantages here over more traditional light sources, such as continuous or sparked short-arc lamps, or conventional lasers. First, the short pulse duration (here $10 \mathrm{ns)} \mathrm{effectively} \mathrm{freezes} \mathrm{the} \mathrm{model} \mathrm{position,}$ removing any motion-related blurring. Second, the light produced is highly monochromatic, meaning that the testgas luminosity that invariably accompanies strong shocks in high-enthalpy facilities can be removed by the insertion of a narrow band-pass filter in the optical path. Finally, compared to conventional lasers, the Cavilux Smart has a very short coherence length, which eliminates the diffraction edges and speckle patterns usually associated with laser-based visualization ${ }^{16}$. In Fig. 4 is shown a sequence of images recorded using a Photron SA-5 camera at a resolution of $448 \times 640$ pixels, highlighting these benefits. This experiment was performed at high-enthalpy $\mathrm{CO}_{2}$ test conditions, for which the test-gas luminosity is significantly more problematic than in the low-enthalpy tests that form the bulk of this study. Images are shown at times corresponding to before the arrival of the flow $(0 \mathrm{~ms})$, during flow establishment $(1.9 \mathrm{~ms})$, removal of the threads $(2.6 \mathrm{~ms})$, the steady test time $(3.5 \mathrm{~ms})$, and the model reaching the limit of motion allowed by the catcher mechanism after the conclusion of the test time (10.0 ms). In all but the first and last images, the bow shock in front of the fore-body is faintly visible; the fitted model outline determined by the tracking algorithm to be discussed shortly is also shown in red. Notably absent are any traces of test-gas luminosity or laser speckle. 


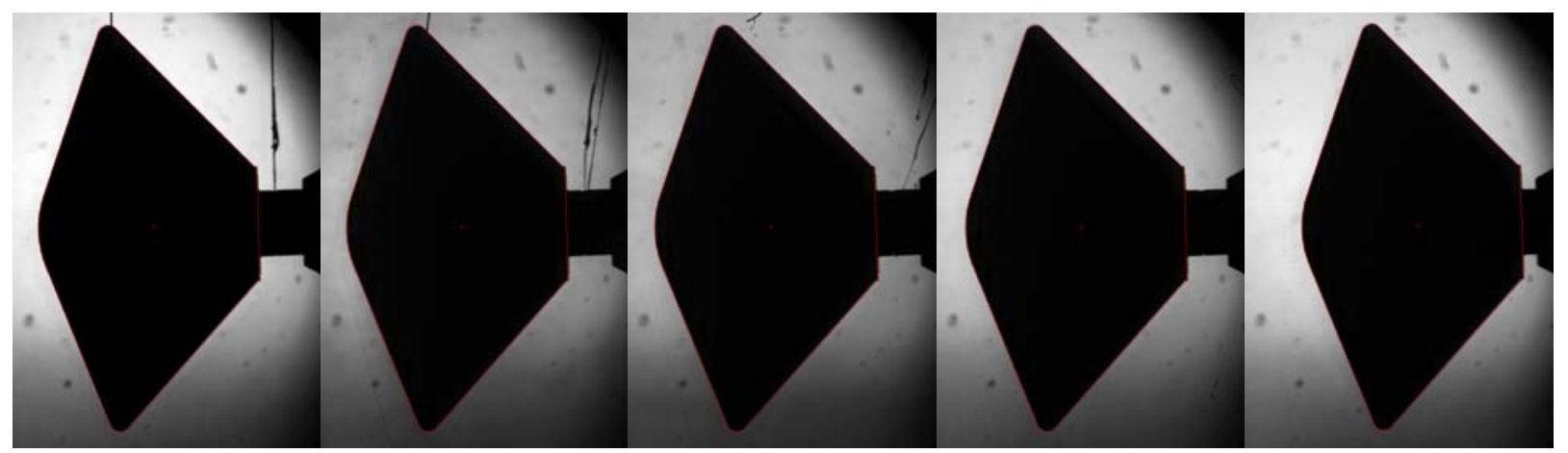

Figure 4. Sequence of focused shadowgraph images showing the development during a typical experiment at times (left to right) $0,1.9,2.6,3.5$, and $10.0 \mathrm{~ms}$ after shock reflection. The fitted model outline is indicated in red in each image.

Two visualization-based tracking techniques were employed, both based upon edge detection and least-squares fitting ${ }^{12}$. The first technique, referred to as analytic-fitting, is substantially identical to that developed in Ref. 10 . The basic procedure is as follows. For each image in the recorded sequence, a pixel-resolution Canny edge detection is carried out, and the edge points lying on the model outline are selected using a semi-automated edge-tracing algorithm. The selected points are then reprocessed with a subpixel-resolution edge detector. Using these points and the known analytical description of the model cross-section, the best-fit values (in the least-squares sense) of the model center-of-geometry position, pitch angle, and the scaling factor between image and physical dimensions are calculated iteratively. From the displacement profiles thus determined, average accelerations are derived by fitting quadratic polynomials over the time period of interest, or unsteady acceleration are obtained through numerical differentiation (with smoothing to reduce the resulting noise).

The second technique, referred to as edge-tracking, is described in Ref. 12. The steps as far as the subpixel edge detection are shared with analytic-fitting. Instead of fitting the model profile in each image, however, (in-plane) rotational and translational displacements are calculated relative to a chosen reference image by matching closest edge points in the two images and solving a sequence of least-squares equations derived from considering the possible motions that satisfy the relative edge-point positions. First, a linearized equation is solved to determine the rotation (this version of the technique is referred to as linearized edge-tracking); the calculated rotations are then used to solve a linear equation for the $x$ and $y$ translations. The accelerations are derived from the displacement profiles in the same manner as in the analytic fitting case.

In Ref. 10, several (at that point unaddressed) sources of uncertainty in the visualization-based tracking technique were identified: motion-related blurring, test-gas luminosity, optical distortions, and structural vibrations. One of the goals of the present experiments was to quantify and/or eliminate these errors. The problems of motionrelated blurring and luminosity were addressed by the choice of light source, as already detailed. To correct for optical distortions present in the imaging system (in particular, astigmatic aberration), a reference "grid", consisting of a Perspex sheet precision-machined with circular dimples at regular intervals, was placed in the test section and visualized with the setup employed for the experiments. The positions of the dimples in the images were determined using the analytic-fitting technique just described, and third-order polynomial transformations between image and physical coordinates were thus determined. Within the tracking routine, these transformations were applied to the model edge points prior to least-squares fitting. The reduction in the profile distortion allowed by this procedure is shown in the left plot of Fig. 5. Here the error in the determined radial position of edge points (i.e., the difference between the radial location of the detected points and the values expected from the fitted analytical cross-sectional profile) is plotted against the internal angle for a typical image recorded with the Shimadzu camera. The use of the distortion correction allows a roughly four-fold decrease in the RMS error, from $0.18 \mathrm{~mm}(0.23$ pixels $)$ to $0.05 \mathrm{~mm}$ ( 0.06 pixels). In terms of accelerations, the effect of the optical distortion on the drag was found to be entirely negligible, with the maximum difference between values derived from corrected and uncorrected edge points being $0.06 \%$. Due to the small angles of attack considered, and the resulting low lift-drag ratios, the percentage differences in the lift accelerations were somewhat larger: up to $2.6 \%$. However, in all cases this was well below the uncertainty due to the precision limits in the tracking technique. We thus conclude that further improvements in the distortion correction are unlikely to offer meaningful benefits to the overall experimental uncertainty. 

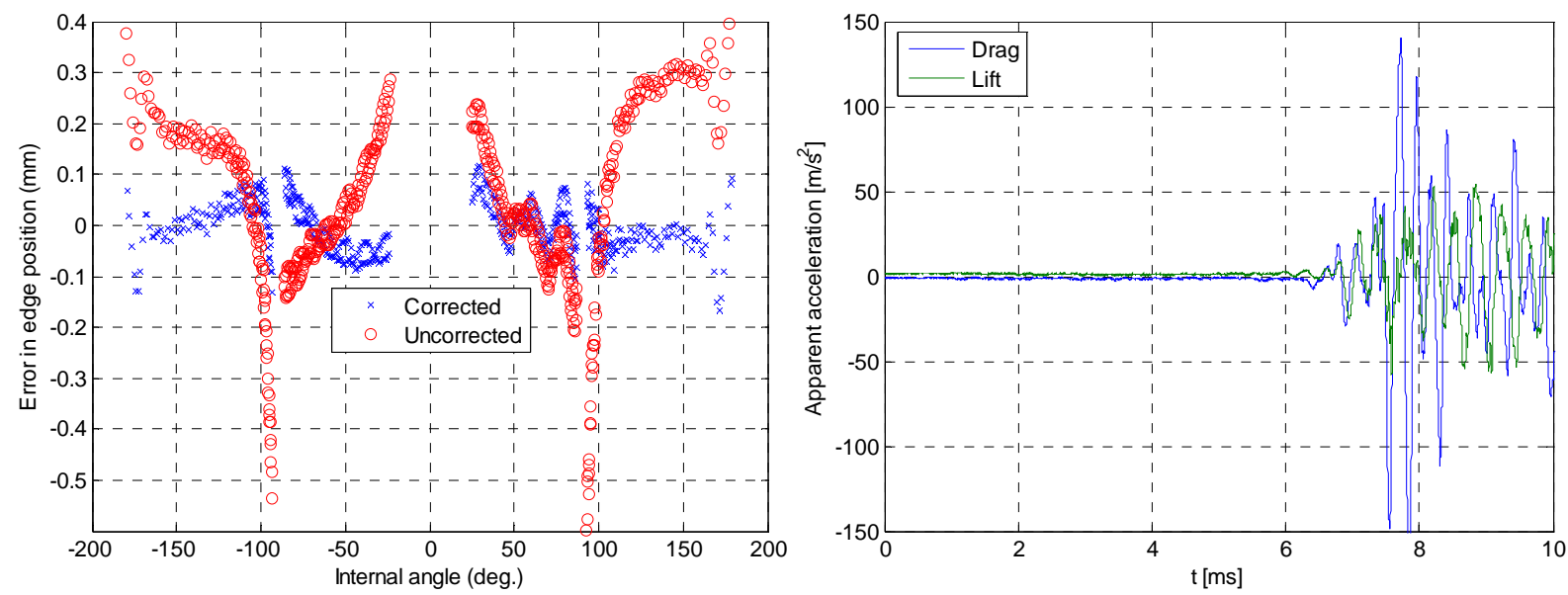

Figure 5. (Left) Errors in the detected edge-point positions (i.e., deviation of the determined point radii from the analytic values) as a function of the internal model angle for profiles both uncorrected and corrected for optical distortions. (Right) Apparent model accelerations due to movement of the camera, as measured by attached accelerometers.

A further source of uncertainty noted in Ref. 10 is the influence of structural vibrations produced by the firing of the facility: these could be transmitted through the optical table to the camera and result in spurious acceleration signals. To quantify this effect, accelerometers were attached to the Shimadzu camera and their outputs were recorded during several experiments. An example is shown in the right plot of Fig. 5: here the apparent acceleration (i.e., taking into account the image magnification) resulting from the camera motion is plotted against the experimental time. The magnitude of the signals until approximately $6 \mathrm{~ms}$ is entirely negligible; the large accelerations visible hereafter were determined to arise from the closing of the camera shutter following the recording of the image sequence, rather than originating from the facility. At least for the present facility then, structural vibrations appear not to be a factor.

\section{Results and Discussion}

An example of a trajectory measured using the analytic-fitting technique is shown in Fig. 6; here, the displacements in the drag and lift directions, as well as the measured pitching angle, are plotted against the experimental time. In each plot, the quasi-steady test period and the quadratic polynomial of best fit to the points during this time are indicated in solid black lines. The model is seen to move approximately 17 and $1.7 \mathrm{~mm}$ in the drag and lift directions, respectively, during the total measurement time of $6.4 \mathrm{~ms}$; the movements during the steady test time are approximately 7 and $0.7 \mathrm{~mm}$, respectively. The pitch angle, which is initially $6^{\circ}$, has decreased by less than $0.4^{\circ}$ after $6.4 \mathrm{~ms}$ and changes by approximately $0.15^{\circ}$ during the test time. The minimum measurement noise relative to the total motion is clearly in the drag profile; the maximum is in the pitch measurement. By calculating the residuals to the quadratic fits shown, measurement precisions of $4.3 \mu \mathrm{m}, 3.3 \mu \mathrm{m}$ and $0.0032^{\circ}$ were estimated for the drag and lift displacements and the pitch angle, respectively. 

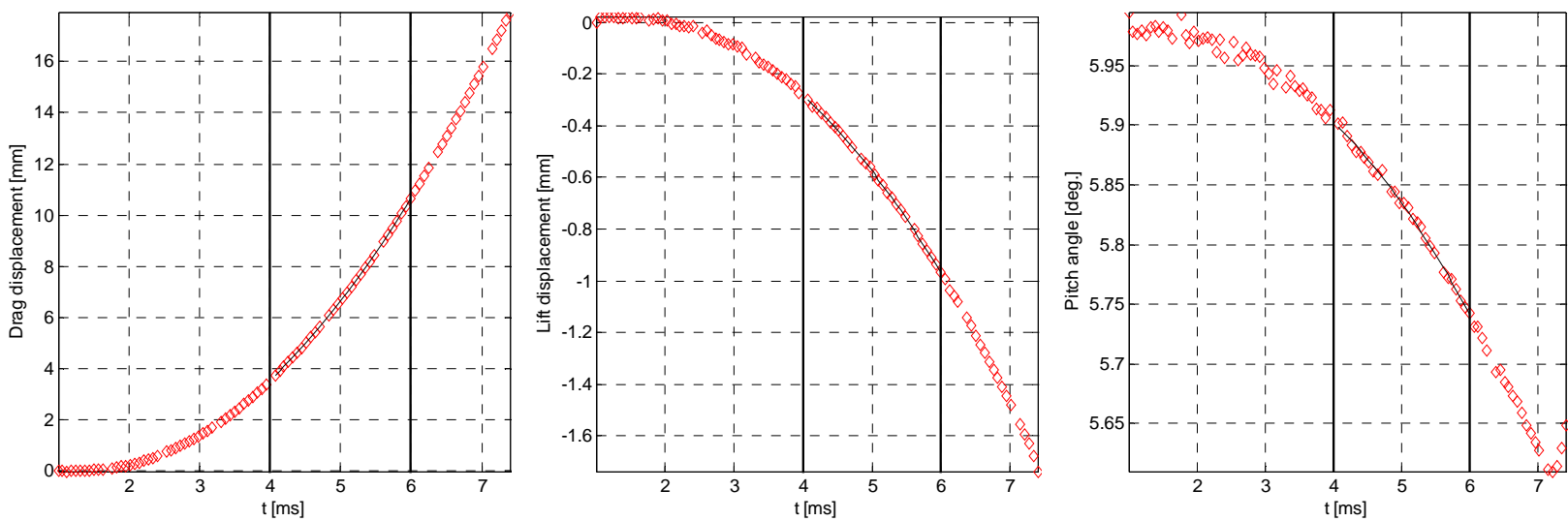

Figure 6. Displacement profiles (drag, lift and pitching angle) as determined by the visualization-based analytic-fitting routine for an experiment in the present investigation. The steady test time and the quadratic polynomial of best fit during this period are indicated in each case by solid black lines.

Considering now the accelerometer measurements, in the left plot of fig. 7 we show the power spectra of two drag accelerometer signals from one experiment. The spectra decay steadily until approximately $4 \mathrm{kHz}$; finiteelement simulations of the model geometry showed the eigenmodes of the model associated with oscillations of the capsule forebody to begin from roughly this point. Thus, to remove the frequency components associated with model oscillations, all accelerometer signals are passed through a 6-pole Butterworth low-pass filter with a $2 \mathrm{kHz}$ cutoff. In the right plot of fig. 7, we compare time-resolved drag and lift accelerations as measured in the experiment shown in fig. 6. The visual-tracking result (analytic fitting) was obtained using a second-order central-difference approximation to the second derivatives of the displacements, followed by the application of three successive moving-average filters of window-size 11,7 and 3 points, respectively, to remove the noise produced by the numerical differentiation. Overall, excellent agreement between the accelerometer and visual-tracking profiles are observed. The mean drag accelerations during the steady test time are $967 \pm 24 \mathrm{~m} / \mathrm{s}^{2}$ (accelerometers) and $960 \pm 5 \mathrm{~m} / \mathrm{s}^{2}$ (visual-tracking); the mean lift accelerations are $-115 \pm 24 \mathrm{~m} / \mathrm{s}^{2}$ (accelerometers) and $-107 \pm 3 \mathrm{~m} / \mathrm{s}^{2}$ (visual-tracking). Also shown in fig. 7 is the measured Pitot pressure, scaled to the level of the drag acceleration. The Pitot pressure and acceleration development are seen to match each other closely until the model reaches the limit of its motion at around $7 \mathrm{~ms}$.
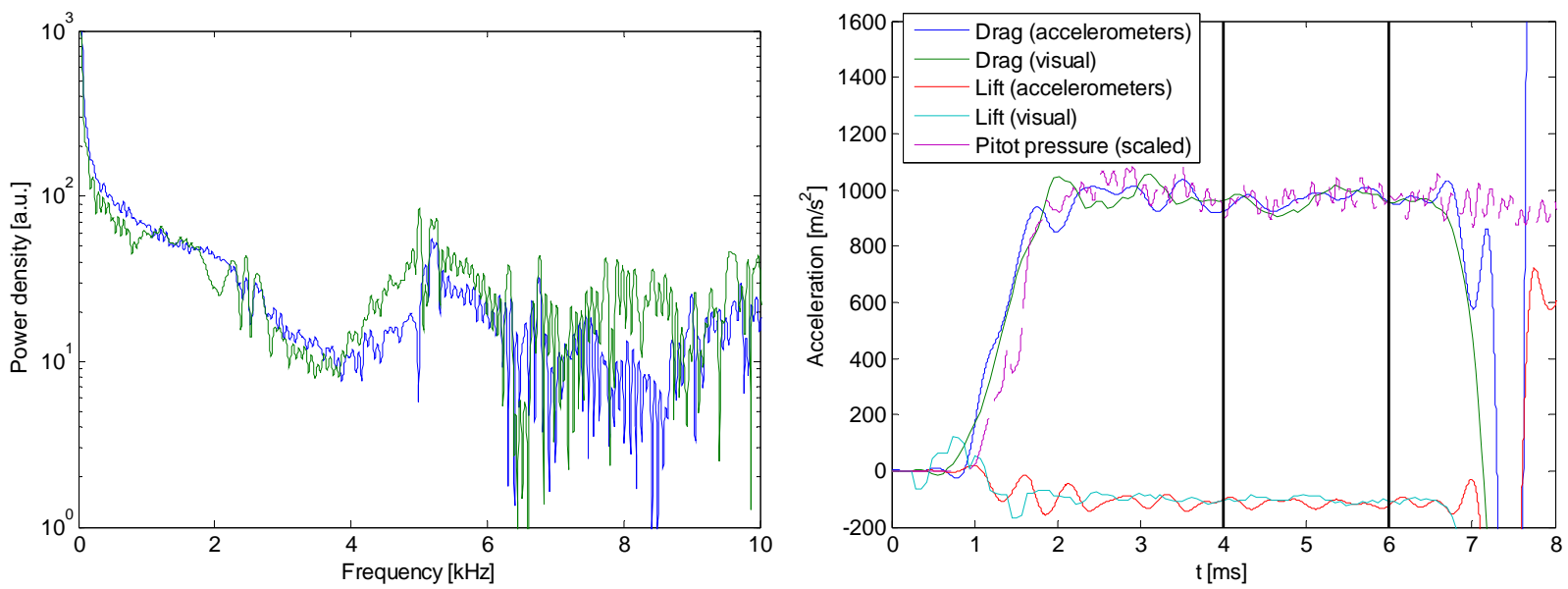

Figure 7. (Lift) Typical power spectra of two drag accelerometers. (Right) Measured time-resolved drag and lift accelerations (from both visual-tracking and accelerometer measurements) and scaled Pitot pressure for a non-zero angle-of-attack experiment.

Mean force coefficients (drag and lift) are plotted versus the capsule angle of attack in fig. 8. The normalization factor here is $1 / 8 \rho_{\infty} u_{\infty}{ }^{2} \pi d^{2}$, where $d$ is the model diameter. The values of $\rho_{\infty} u_{\infty}{ }^{2}$ are derived from the TAU nozzle computation referred to in section II. Shot-to-shot variation is accounted for by using the fact that, to a good approximation, $\rho_{\infty} u_{\infty}{ }^{2}$ is proportional to the Pitot pressure; thus, the value of of $\rho_{\infty} u_{\infty}{ }^{2}$ was scaled by the measured 
Pitot pressure in each experiment, divided by the mean Pitot pressure over all experiments. Plotted in fig. 8 are results from accelerometer measurements, the two visual-tracking techniques, as well as theoretical estimates obtained by assuming a modified Newtonian distribution on the capsule forebody. The error bars in each case indicate only the estimated uncertainty in the measured acceleration, not in the normalization quantity, $\rho_{\infty} u_{\infty}{ }^{2}$; this is to allow a more precise comparison of the measurement techniques within each experiment. For the visualizationbased results, the errors are derived from the residuals to the quadratic fits to the displacement curves, as outlined in Ref. 10. For the accelerometer measurements, the plotted error is simply the standard deviation in the mean value during the test time (considering that the frequency content above $2 \mathrm{kHz}$ has been removed from these signals, this is only an indication of the low-frequency variation of the accelerations, rather than a rigorous estimate of the uncertainty). The standard deviation in the measured Pitot pressure (and thus, by extension, in $\rho_{\infty} u_{\infty}{ }^{2}$ ) during the steady test time was typically around 3.5\%; for reference, the symbol-less error bars to the extreme right of each plot in fig. 8 show the corresponding variation in the force coefficients at the levels indicated.
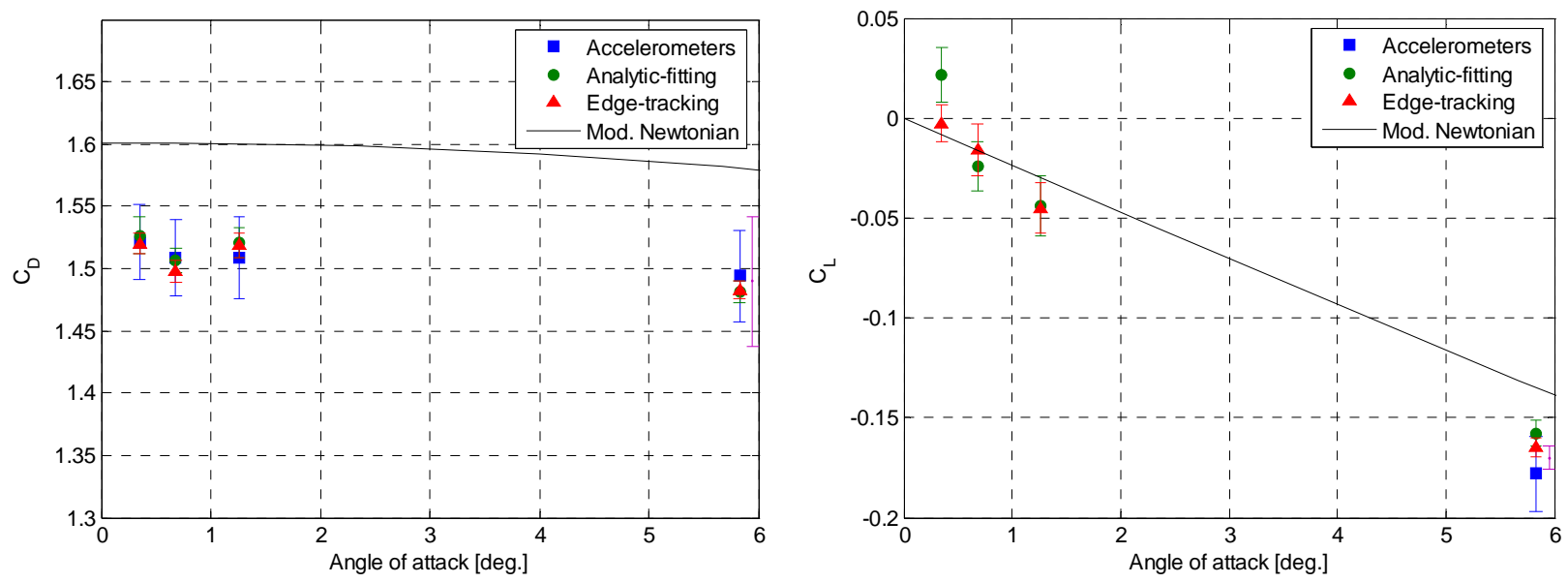

Figure 8. Measured drag (left) and lift (right) coefficients versus the angle of attack for the present experiments; the error bars in each case indicate the error only in the acceleration measurement. Curves indicating the normalized forces assuming a modified Newtonian pressure distribution on the forebody are also shown. The symbol-less error bars to the extreme right of each plot shows the percentage variation in the measured Pitot pressure, scaled to the corresponding value of the force coefficient.

Overall, agreement between the measurement techniques is very good. All results agree to within the measurement uncertainty, with the sole exception of the visualization-based lift results at the smallest angle of attack considered. The edge-tracking value is more physically realistic here, suggesting that this technique may be slightly preferable to analytic fitting. Note that accelerometer-based lift measurements were only available at the largest angle of attack. The theoretical curves show good qualitative agreement with the measured trends, but the drag is overestimated by approximately $5 \%$ and the magnitude of the lift coefficient is underestimated. In general, however, the modified Newtonian result is seen to be capable of adequate predictions at these test conditions. In the case of the drag coefficient, the variation in $\rho_{\infty} u_{\infty}{ }^{2}$ clearly dominates the errors in the visualization-based acceleration measurements. At angles of attack close to zero, the small value of the lift coefficient means that the acceleration error dominates the overall uncertainty; however, if the angle of attack is increased to around $6^{\circ}$, the variation in $\rho_{\infty} u_{\infty}{ }^{2}$ becomes comparable to the acceleration error. This situation can only be expected to improve as new camera technology allows higher resolutions and frame-rates to be employed.

In fig. 9 are shown pitching accelerations measured with the various techniques. Again, agreement to within the measurement errors are obtained in all cases, but this may be due in part to the large uncertainties. As noted in Ref. 10 , accurate pitching measurements on blunt geometries with visualization-based techniques are difficult, as the change in angle relative to the angular precision is typically small compared to the translational components. This was especially the case for the experiments at smaller angles of attack in the present campaign, in which the precision was limited to around $0.007-0.009^{\circ}$ in comparison to $0.003^{\circ}$ for the larger angle of attack (for which the indicated error is less than $10 \%$ of the mean value). The origin of this decreased precision is not clear. Assuming a precision of approximately $0.003^{\circ}$ can be obtained, however (this should be quite achievable with increased image resolution: the angular precision in the sequence shown in fig. 4 was estimated at around $0.001^{\circ}$, for example), visualization-based techniques appear capable of higher accuracy than accelerometer-based measurements. 


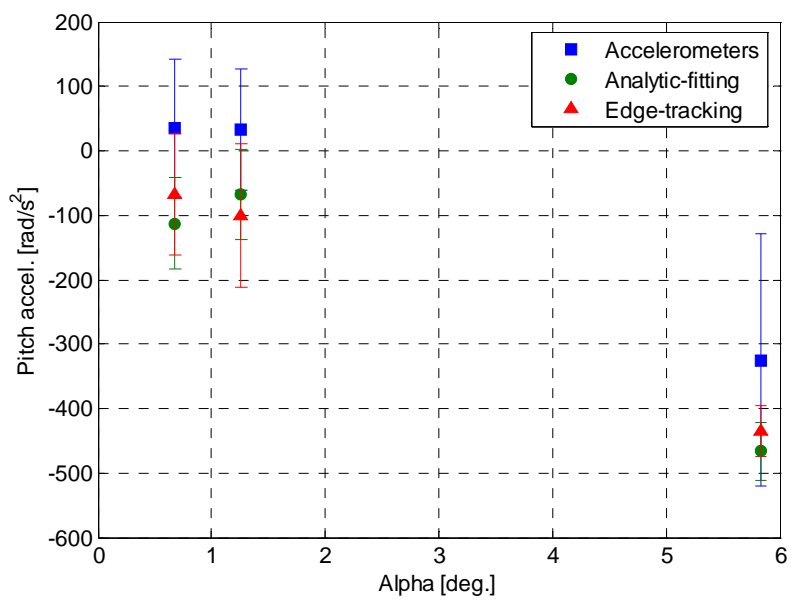

Figure 9: Measured pitching accelerations versus angle of attack for the various techniques.

\section{Acknowledgments}

The authors gratefully acknowledge the assistance of the HEG technical staff, in particular Ingo Schwendtke, Mario Juenemann and Sarah Trost, in preparing the experiments.

\section{References}

${ }^{1}$ Bernstein, L., "Force Measurement in Short Duration Hypersonic Facilities," AGARDograph No. 214, 1975.

${ }^{2}$ Sanderson, S. R., and Simmons, J. M., "Drag Balance for Hypervelocity Impulse Facilities,” AIAA Journal, Vol. 29, No. 12, 1990, pp. 2185-2191.

${ }^{3}$ Marineau, E., "Force Measurements in Hypervelocity Flows with an Acceleration Compensated Piezoelectric Balance," Journal of Spacecraft and Rockets, Vol. 48, No. 4, 2011, pp. 697-700.

${ }^{4}$ Tanno, H., Itoh, K., Saito, K., Abe, A., and Takayama, K., "Unsteady Force Measurement in Shock Tubes," Review of Scientific Instrumentation, Vol. 75, No. 2, 2004, pp. 532-536.

${ }^{5}$ Tanno, H., Kodera, M., Komuro, T., Sato, K., Takahashi, M., and Itoh, K., “Aerodynamic Force Measurement on a LargeScale Model in a Short-Duration Test Facility," Review of Scientific Instrumentation, Vol. 76, No. 3, $2005,035107$.

${ }^{6}$ Warren, W. R., Kaegi E. M., and Geiger, R. E., "Shock Tunnel Experimental Techniques for Force and Moment and Surface Flow Direction Measurements," ARS Journal, Vol. 31, No. 1, pp. 82-83.

${ }^{7}$ Horstmann, C. C., and Kussoy, M. I., "Free Flight Measurements of Aerodynamic Viscous Effects on Lifting Re-entry Bodies," Journal of Spacecraft, Vol. 4, No. 8, 1967, pp. 1064-1069.

${ }^{8}$ Tanno, H., Sato, K., Komuro, T., Takahashi, M., Itoh, K., Fujita, K., Laurence, S., Hannemann, K., "Force Measurement Using Non-restrained Models in a Shock Tunnel," $7^{\text {th }}$ ESA Aerothermodynamics Symposium, Brugge, Belgium, 9-12 May, 2011

${ }^{9}$ Laurence, S. J., and Hornung, H. G., "Image-Based Force Measurement in Hypersonic Facilities," Experiments in Fluids, Vol. 46, 2009, pp., 343-353.

${ }^{10}$ Laurence, S. J., and Karl, S., "An Improved Visualization-Based Force-Measurement Technique for Short-Duration Hypersonic Facilities," Experiments in Fluids, Vol. 48, 2010, pp., 949-965.

${ }^{11}$ Robinson, M., and Hannemann, K., "Short Duration Force Measurements in Impulse Facilities", 25th AIAA Aerodynamic Measurement Technology and Ground Testing Conference, San Francisco, CA, 5-8 June, 2006

${ }^{12}$ Laurence, S. J., "On tracking the motion of rigid bodies through edge-detection and least-squares fitting," Experiments in Fluids, Vol. 52(2), 2012, pp., 387-401.

${ }^{13}$ Hannemann, K., Schnieder, M., Reimann, B., and Martinez Schramm, J., "The influence and delay of driver gas contamination in HEG", 21st AIAA Aerodynamic Measurement Technology and Ground Testing Conference, Denver, CO, 19-22 June, 2000

${ }^{14}$ Hannemann, K., "High Enthalpy Flows in the HEG Shock Tunnel: Experiment and Numerical Rebuilding”, 41st AIAA Aerospace Sciences Meeting and Exhibit, 6-9 Jan, Reno, Nevada, 2003

${ }^{15}$ Gerhold, T., Friedrich, O., Evans, J., Galle, M., "Calculation of Complex Three-Dimensional Configurations Employing the DLR-TAU-Code", AIAA-Paper 97-0167, 1997

${ }^{16}$ Settles, G., "Schlieren and Shadowgraph Techniques," Springer, New York, 2006 